\title{
Perfil dos profissionais de saúde e sua relação com a Síndrome de Burnout: uma revisão narrativa
}

\author{
Profile of health professionals and their relationship to Burnout Syndrome: a narrative review
}

Perfil de los profesionales de la salud y su relación con el Síndrome de Burnout: una revisión narrativa

Carlos Humbertto Marques Cavalcanti ${ }^{1 *}$, Leide Dayane Barbosa da Silva ${ }^{1}$, José Luiz dos Santos Castilho', Elder Yuji Kimura da Silva², Lívia Almeida Rocha Bandeira², Thiago Rodrigo Fernandes da Silva ${ }^{3}$.

\section{RESUMO}

Objetivo: Realizar uma análise narrativa com base em literaturas científicas sobre o entendimento da Síndrome de Burnout e o perfil dos profissionais de saúde afetados gerando consequências na saúde desses indivíduos no seu âmbito biopsicossocial. Revisão bibliográfica: A Síndrome de Burnout (SB) é um conjunto de sinais e sintomas que levam a uma profunda exaustão intelectual e corporal devido exposição significativa a momentos que necessitam de certa carga emocional para lidar com as demandas advindas do meio profissional. Nesse contexto, os profissionais de saúde são submetidos a carga de estresse tanto físicas quanto psicológicas, os que atuam no atendimento de urgência e emergência podem ter níveis mais elevados de Burnout, isso ocorre devido à pressão exigida em seu cargo. Por conseguinte, a exaustão dos trabalhadores gera complicações também aos pacientes, já que a qualidade do atendimento pode ser prejudicada. Outrossim, indivíduos casados ou em união estável apresentam maior resistência aos problemas emocionais justificados pela experiência da responsabilidade familiar maior. Considerações finais: Verificase que há uma vasta literatura sobre esse assunto, entretanto há limitação nas amostras que implica em resultados fidedignos para traçar o perfil de um profissional suscetível à SB.

Palavras-chave: Síndrome do esgotamento, Esgotamento profissional, Esgotamento psicológico, Estresse profissional.

\begin{abstract}
Objective: To carry out a narrative analysis based on scientific literature on the understanding of Burnout Syndrome and the profile of health professionals affected generating consequences on the health of these individuals in its biopsychosocial scope. Bibliographic review: Burnout Syndrome (BS) is a set of signs and symptoms that lead to profound intellectual and bodily exhaustion due to significant exposure to moments that require a certain emotional charge to deal with the demands arising from the professional environment. In this context, health professionals are subjected to a load of stress both physical and psychological, those who work in urgent and emergency care may have higher levels of Burnout, this is due to the pressure required in their position. Therefore, workers exhaustion also causes complications for patients, as the quality of care can be impaired. Furthermore, individuals who are married or in a stable relationship are more resistant to emotional problems justified by the experience of greater family responsibility. Final considerations: It appears that there is a vast literature on this subject, however there is a limitation in the samples that implies reliable results to outline the profile of a professional susceptible to BS.
\end{abstract}

Keywords: Burnout syndrome, Professional Burnout, Psychological Burnout, Professional stress.

\footnotetext{
${ }^{1}$ Faculdade Ages de Medicina (FAM), Jacobina - BA. *E-mail: carlos.humbertto10@hotmail.com

2 Universidade Federal da Grande Dourados (UFGD), Dourados - MS.

${ }^{3}$ Universidade Federal de Minas Gerais (UFMG), Belo Horizonte - MG.
} 


\section{RESUMEN}

Objetivo: Realizar un análisis narrativo basado en literatura científica sobre la comprensión del Síndrome de Burnout y el perfil de los profesionales de la salud afectados, generando consecuencias sobre la salud de estos individuos en su ámbito biopsicosocial. Revisión bibliográfica: El Síndrome de Burnout es un conjunto de signos y síntomas que conducen a un profundo agotamiento intelectual y corporal debido a la exposición significativa a momentos que requieren cierta carga emocional para hacer frente a las demandas derivadas del entorno profesional. En contexto, los profesionales de la salud están sometidos a una carga de estrés físico y psicológico, quienes laboran en atención de urgencias y emergencias pueden tener niveles más altos de Burnout, por la presión que se requiere en su puesto. El agotamiento de los trabajadores también causa complicaciones a los pacientes, ya que la calidad de la atención puede verse afectada. Además, las personas casadas o en relación estable son más resistentes a los problemas emocionales justificados por la experiencia de una mayor responsabilidad familiar. Consideraciones finales: Existe una amplia literatura sobre este tema, aún existe una limitación en las muestras que implica resultados confiables para perfilar el perfil de un profesional susceptible de BS.

Palabras clave: Síndrome de Burnout, Agotamiento profesional, Agotamiento psicológico, Estrés laboral.

\section{INTRODUÇÃO}

A Síndrome de Burnout (SB) é conceituada como um conjunto de sinais e sintomas que levam a uma profunda exaustão intelectual e corporal motivada pela exposição significativa a momentos que necessitam de certa carga emocional para lidar com as demandas advindas do meio profissional. Nesse contexto, o esgotamento profissional é consequência de um excesso físico e mental que leva o indivíduo à exaustão, por conseguinte afetando os relacionamentos, o ambiente de trabalho e provocando um clima desumano e impessoal, com o tempo o indivíduo sente-se insatisfeito profissionalmente e apático frente ao futuro (CÂNDIDO J e SOUZA LR, 2017).

Assim, a síndrome do esgotamento profissional foi definida pelo psiquiatra Herbert Freudenberger, em 1974, o qual percebeu a exaustão, decepção e perda de interesse dos profissionais da saúde em decorrência do desempenho de suas funções e a partir de Christina Maslach foram acrescentados o distanciamento social e as consequências da Síndrome de Burnout como uma resposta a fontes crônicas de estresse emocional e interpessoal no trabalho (ALVES ME, 2017).

Frequentemente, os profissionais de saúde estão expostos a inúmeros riscos em suas atividades laborais, como riscos físicos, biológicos, psicossociais, entre outros. Portanto, estes trabalhadores estão sujeitos a adoecimento relacionado ao trabalho e são encontrados diversos sintomas relacionados ao burnout: fisicamente, o indivíduo pode apresentar fadiga, dores musculares, cefaleias, alterações gastrointestinais e/ou cardiovasculares, distúrbios do sistema respiratório e do sono, além de disfunções sexuais; já relacionado com as alterações psíquicas, tem-se falta de concentração, baixa autoestima, sintomas depressivos e ansiosos (FAGUNDES PS, 2016).

Desse modo, é importante identificar tais manifestações e o estado de acometimento em que o profissional se encontra para melhor construção de um plano de cuidado voltado aos aspectos biopsicossociais. Os estudos demonstram que a Síndrome de Burnout traz ao trabalhador prejuízos pessoais e também no trabalho promovendo baixo rendimento e danos irremediáveis, podendo levar ao abandono do emprego (FAGUNDES PS, 2016).

Além disso, a exposição ao estresse é fundamental para o desencadeamento de diversos problemas psíquicos, uma vez que o estresse promove uma modificação no estado do organismo, visto que desencadeia um complexo de respostas fisiológicas e emocionais. Dessa maneira, muitas demandas de trabalho provocam certa carga de estresse acarretando alterações mentais, dentre essas requisições: atividades extemporâneas, tarefas com tempo estipulado, interrupção das atividades e conflitos hierárquicos (SILVA GN, 2019; MOREIRA $H A$, et al., 2018). 
Ademais, em relação a equipe de saúde pode-se citar algumas circunstâncias específicas que corroboram para diminuição na qualidade de vida, como: plantões noturnos, contraturno, dupla jornada de trabalho e a desvalorização profissional. Essas condições favorecem o desgaste profissional, já que situações de trabalhos precários predispõem os trabalhadores de saúde a condições de adoecimento em situações superiores às das pessoas a que prestam seus serviços (BARRETO GAA, et al., 2021; COSTA KNFM, et al., 2017).

Diante do exposto, o presente estudo tem como objetivo realizar uma análise narrativa com base em literaturas científicas sobre o entendimento da Síndrome de Burnout e o perfil dos profissionais de saúde afetados gerando consequências na saúde desses indivíduos no seu âmbito biopsicossocial.

\section{REVISÃO BIBLIOGRÁFICA}

Existem muitas afecções que podem acometer os trabalhadores, torna-se possível sua análise na lista de doenças relacionadas a atividades laborais, em que constam 198 patologias, entre elas o Burnout ou Síndrome do Esgotamento Profissional. É importante ressaltar que a SB é um feedback aos estressores emocionais e interpessoais crônicos no trabalho, está inserido na Classificação Estatística Internacional de Doenças e Problemas Relacionados à Saúde, CID-10, possui um código específico de identificação, Z73.0, e está relacionada a uma desordem no modo de vida dos indivíduos (PANTOJA FGB, et al., 2017; MOREIRA HA, et al., 2018).

Além disso, a Síndrome de Burnout (SB) apresenta três elementos fundamentais, sendo eles: a Exaustão Emocional (EE), Despersonalização (DP) e Redução da Realização Pessoal (RP). Para avaliação desses elementos, o instrumento comumente utilizado é o Maslach Burnout Inventory (MBI), que apresenta como criadores Maslach e Jackson, atualmente é considerado um instrumento fidedigno devido sua complexidade em avaliar as diferentes características que são expressas pela SB em comparação a outros tipos de questionários, além de ser instrumento para avaliar como os profissionais vivenciam a sua rotina. Portanto, 0 MBI trata-se de um questionário contendo 22 questões sobre sentimentos em relação à atividade laboral do profissional e os sintomas apresentados, bem como sua frequência (MOREIRA HA, et al., 2018; BRAYDE DHS e COSTA TNM, 2019).

Outrossim, existem questões específicas de avaliação do indivíduo para os três elementos centrais que compõem a síndrome (EE, DP e RP). Cada elemento possui seu próprio número de questões e para essas é atribuído um determinado conceito, podendo ser: baixo, médio e alto. Desse modo, para a EE são 9 questionamentos, relacionado a DP são 5 questionamentos e RP são 8 questionamentos (MOREIRA HA, et al., 2018; SILVEIRA ALP, et al., 2016).

A síndrome está relacionada com inúmeros processos danosos à saúde do indivíduo, notadamente à saúde emocional, com características como irritabilidade; além do risco de progredir para humor depressivo; cansaço; redução da autoestima; pensamentos suicidas; alterações cognitivas; mialgia; distúrbios do sono e sexuais; comprometimento imunológico, cardiovascular, hormonal e isolamento social (LIMA AS, et al., 2018).

À vista disso, a SB não só afeta o indivíduo, mas também a sua corporação, as tarefas realizadas por ele e a sociedade, uma vez que um profissional com sintomas de desgastes físicos e emocionais são mais propensos a terem um rendimento negativo com altas taxas de erros em procedimentos executados, negligência e aumento de conflitos familiares e sociais (SILVEIRA ALP, et al., 2016).

A SB causa transtornos de forma bilateral no trabalho, tanto para quem presta o serviço como para quem contrata o funcionário. É válido salientar que sobrecarga de tarefas, desmotivação e gestão autoritária pode acarretar absenteísmo, impactando de forma negativa na produção da empresa. Outrossim, existem três características que o ambiente de trabalho deve adotar que são essenciais para que os trabalhadores cheguem à satisfação profissional: o reconhecimento, o sentimento de importância e a valorização dos funcionários. Por conseguinte, um ambiente de trabalho que não oferece recursos para seu funcionamento e não busca a valorização do indivíduo caracteriza-se como aspecto para o desenvolvimento da SB (LIMA AS, et al., 2018). 
Paralelamente, a promoção da integralidade no cuidado é de suma relevância para agir no complexo saúde-doença, visto que o termo indivíduo saudável não se caracteriza apenas pela ausência de afecções. Nesse contexto, a saúde mental apresenta destaque por ser um pilar importante na manutenção de um indivíduo hígido, pois manifesta relevância na avaliação do bem-estar e o seu desequilíbrio apresenta impactos tanto no indivíduo como na sociedade em que ele está inserido. É importante salientar que os indivíduos estão englobados pela sua rotina, com inquietações, ansiedades, angústias e preocupações que provocam sofrimento psicossomático. Portanto, esse desequilíbrio leva ao desgaste emocional culminando em sintomas causados pelo esgotamento de não atender as expectativas perante a realidade (ALVES ME, 2017).

A incidência da Síndrome de Burnout está ligada a diversos fatores, entre eles, a forma como o estresse emocional é ligado aos problemas diários e como as pessoas lidam com a convivência do meio social, portanto alguns estudos traçaram o perfil dos profissionais suscetíveis à SB, com destaque para a profissão, notadamente medicina; estado civil, apresentar duas ou mais atividades laborais, gênero feminino, além do campo de atuação. Nesse contexto, compreender o perfil de uma afecção que assola o indivíduo é essencial para a tomada de decisões no combate dessa problemática (SILVEIRA ALP, 2016).

Portanto, há uma prevalência do número de médicos com a presença de cansaço emocional estressante, uma vez que eles lidam com um meio social cada vez mais complexo, com mudanças de informações e adaptações na forma de lidar com as patologias (ROCHA APF, et al., 2015).

Segundo dados de pesquisa sistemática, a SB possui prevalência nas profissões de natureza assistencial, incidente em 1 a cada 2 médicos, a nível mundial. Na medicina, a SB é notada em níveis altos em mais de $20 \%$ dos médicos brasileiros, em amostra envolvendo quase 8 mil profissionais de todos os estados brasileiros. As cinco especialidades com maior prevalência de casos de SB são: medicina de Unidade de Terapia Intensiva- UTI, medicina de família- MF, medicina de emergência, medicina interna e ortopedia, portanto compreender os grupos suscetíveis ao desenvolvimento dessa patologia é chave mestra nas devidas intervenções. Assim, percebe-se a existência de fatores agravantes e atenuantes que contribuem para 0 surgimento ou não da síndrome (MOREIRA HA, et al., 2018).

É importante salientar que a SB tem destaque em outras categorias profissionais. Segundo estudo realizado em um hospital infantil, localizado em Campinas/SP, no qual três categorias profissionais foram analisadas: médicos, enfermeiros e técnicos de enfermagem. Os médicos apresentaram uma porcentagem mais elevada de desgaste emocional e sentiam realização profissional baixa. Já os enfermeiros se destacaram por uma alta taxa de despersonalização e os técnicos de enfermagem apresentaram um maior nível de correlações com variáveis biopsicossociais (ZANATTA AB e LUCCA SR, 2015).

Nesse contexto, os profissionais de saúde estão submetidos a uma carga de estresse que ocasiona o surgimento de síndromes com consequências tanto físicas como psicológicas, em especial, por atuar em um local que requer um ritmo ainda mais acelerado e intenso. Dessa forma, precisam ter mais agilidade e atenção para atender as demandas diárias interligadas a exaustiva carga horária e condições de trabalho. Apesar de serem capacitados para lidar com situações de forte carga emocional, isso não os deixa isentos de apresentar desequilíbrio emocional, notadamente altos níveis de estresse. Por conseguinte, prejudicando na qualidade da assistência ofertada aos pacientes (SANTOS JS, et al., 2018; TEIXEIRA GS, et al., 2019).

Paralelamente, a gênese da SB ocorre de maneira lenta e gradativa no profissional de saúde. Apresentase em três etapas, a primeira se relaciona com aumento das atividades em detrimento aos recursos humanos e materiais, a segunda etapa está relacionada com a fase de adaptação à sobrecarga laboral, essa fase é perceptível sinais e sintomas como fadiga, esgotamento físico e emocional e, por fim, na terceira etapa, temse o distanciamento emocional (PÊGO FPL e PÊGO DR, 2016).

É possível observar através dos artigos a importância do aprofundamento sobre a SB principalmente devido seus impactos na saúde do trabalhador. Desse modo, a capacidade que os indivíduos tendem a superar as dificuldades é um fator de extrema relevância que deve ser considerado para a avaliação da SB, 
visto que a habilidade do trabalhador em conseguir alcançar o estado de resiliência, com ênfase nos fatores pessoais, está relacionada a uma medida preventiva para o não acometimento de síndromes de esgotamento mental (TOMAZ HC, et al., 2020).

A partir disso, os profissionais da saúde estão mais suscetíveis a possuírem cansaço exaustivo, pois na sua rotina necessitam diariamente de uma carga emocional excessiva para lidarem com os problemas, conseguirem cumprir metas e realizarem plantões. Dessa maneira, a transição epidemiológica das enfermidades que afetam o indivíduo e como os profissionais lidam com o processo de saúde-doença rotineiramente alinhada à desvalorização profissional influencia diretamente no âmbito logístico e psicológico dos trabalhadores, uma vez que a exigência de um raciocínio clínico mais eficaz voltado para inovações no modo de agir e trabalhar em conjunto faz com que os profissionais de saúde se cobrem cada vez mais necessitando de uma certa carga emocional gradativamente maior para lidar com as demandas do cotidiano (ZOMER F e GOMES KM, 2017).

Além da categoria profissional, outro fator de vulnerabilidade à SB é a relação do estado civil. Conforme estudos, os viúvos estão relacionados com nível alto de exaustão emocional, já os separados apresentam nível baixo. Outrossim, indivíduos casados ou em união estável apresentam maior resistência aos problemas emocionais justificados pela experiência da responsabilidade familiar maior. Entretanto, aos divorciados, a menor exaustão emocional no trabalho está relacionada pela menor carga emocional familiar, uma vez que os problemas conjugais interferem sobremaneira no desenvolvimento profissional, entretanto uma vez superados através da reconciliação ou separação permitem menor propensão à SB (CRUZ SP e ABELLÁN MV, 2015).

É importante ressaltar ainda que os solteiros apresentam risco para SB. Conforme pesquisa realizada em Hospital Universitário no Belém, Pará, com 62 trabalhadores houve uma prevalência maior de SB no grupo dos solteiros, uma vez que $72 \%$ destes manifestou burnout em pelo menos uma dimensão crítica, ao passo que somente $32 \%$ do grupo dos casados/união estável apresentou prejuízo em uma das esferas da síndrome. Além disso, no grupo de entrevistados que exerciam duas ou mais atividades remuneradas, $48,6 \%$ apresentaram a síndrome, contra $27 \%$ de prevalência no grupo que exercia somente uma atividade. Por fim, foi observado maior prevalência de SB no grupo de melhor remuneração, visto que $78,6 \%$ dos que recebiam mais de 10 salários-mínimos apresentaram a síndrome, contra $45 \%$ do grupo que recebia até 4 saláriosmínimos (PANTOJA FGB, et al., 2017).

A partir dos estudos foi realizada uma análise de genitores e a SB. Sabe-se que muitos profissionais possuem filhos e precisam disponibilizar tempo para os cuidados deles. No entanto, quando é feita uma associação entre possuir filho ser um fator de risco aumentado para SB não fica evidenciado essa questão, visto que não existe associação significativa com a presença ou a ausência de filhos para o desenvolvimento da SB. Diante do exposto, fica claro que ainda se faz necessário mais pesquisas em relação à maternidade/paternidade e sua associação ao esgotamento profissional (CRUZ SP e ABELLÁN MV, 2015).

É importante considerar que a literatura presume que um indivíduo com alto grau de escolaridade possui atribuições de maiores responsabilidades e altas expectativas de satisfação profissional em relação à profissão exercida, o que gera maior estresse. Além disso, o trabalho estressante está associado positivamente ao maior risco de SB (BALDONEDO-MOSTEIRO M, et al., 2019). Um fator agravante para isso é a associação de profissionais da saúde com mais de um vínculo empregatício, notadamente a equipe de enfermagem. Nesse contexto, a situação de sobrecarga laboral e a dificuldade de conciliar a dupla jornada podem contribuir com o processo de adoecimento do servidor (ZOMER FB e GOMES KM, 2017).

Outro fator de risco associado à SB é o ambiente de trabalho agravante e o gênero feminino. Segundo estudo realizado em seis Unidades de Terapia Intensiva (UTI) de São Luís, Maranhão, com 60 médicos plantonistas, houve uma prevalência de Burnout em $13,3 \%$ da população pesquisada. No estudo, a SB é mais comum em mulheres, com perfil de menos de 40 anos, com união estável e com formação menor ou igual a 10 anos, está relacionada mais comumente com especialidade em Pediatria, Medicina Intensiva ou Neonatologia (MARQUES GLC, et al., 2018). 
Além disso, outro fator estudado é referente à faixa etária. Percebe-se que os profissionais mais jovens têm uma maior propensão de desenvolver a SB, tal fato pode ser justificado por falta de socialização com os colegas de trabalho, o que dificulta as situações de enfrentamento de problemas (LIMA AS, et al., 2018).

Além disso, há relação dos profissionais que atuam no atendimento de urgência e emergência e níveis mais elevados de burnout. Isso ocorre devido aos diversos fatores estressantes que são típicos desse cenário especializado, dentre eles: os ruídos excessivos na UTI, possibilidade de complicações no atendimento, problemas administrativos e o comprometimento da equipe multidisciplinar. À vista disso, por causa da pressão exigida nesse cargo em que os profissionais precisam se manter calmos e focados em situações que por vezes não tem um planejamento prévio, é necessário ser eficiente, e dominar os procedimentos para que não ocorra erros (MARQUES GLC, et al., 2018).

Além disso, o ambiente de UTI é um local de trabalho estressante, ficando os profissionais responsáveis por equipamentos e manter a vigilância do paciente o que gera, muitas vezes, desequilíbrio emocional afetando diretamente sua qualidade de vida. Nesse contexto, a sobrecarga laboral é um dos fatores causadores de estresse e desmotivação da equipe de saúde. Um fator, observado nos estudos, que agrava a saúde física e mental dos profissionais é o trabalho em regime de plantão e a dupla jornada, onde esse profissional passa a trabalhar excessivamente, por conseguinte irá gerar estresse físico e emocional (ROCHA APF, et al., 2015).

A partir dos estudos analisados foi possível inferir também uma deficiência de trabalhos sobre burnout em profissionais da Atenção Primária à Saúde (APS), principalmente no Brasil. Os trabalhadores da APS também lidam diretamente com o sofrimento humano, tornando-os assim vulneráveis a desenvolver SB. Como esses profissionais estão próximos à comunidade, eles se sentem pressionados e cobrados a dar soluções aos problemas relacionados à saúde da população em que atuam além de existir um déficit no quadro de funcionários, precisam alcançar uma produtividade cada vez maior e por muitas vezes não possuem matérias ou infraestrutura necessária (LIMA AS, et al., 2018).

Ademais, é essencial a disseminação de informações e orientações sobre Burnout e os males que podem causar à pessoa e às instituições. Essas medidas buscam prevenir o estresse causado pela profissão dessas pessoas, o que levará a uma qualidade de vida e um crescimento pessoal e profissional, havendo assim um benefício para a população que será atendida e consequentemente à instituição (ZOMER FB e GOMES KM, 2017).

Portanto, o tema estudado permite uma revisão dos aspectos relacionados à Síndrome de Burnout mostrando a prevalência e incidência na sociedade hodierna. Dessa maneira, a partir dessa observação percebe-se a importância dos fatores sociais para que no futuro sejam voltados elementos para construção de políticas públicas voltadas para essa temática, visto que medidas de precaução auxiliam para que ações possam ser desenvolvidas para minimizar o cansaço mental dos profissionais de saúde corroborando para a satisfação profissional e o bem-estar no convívio social (SILVA RAD, 2018).

\section{CONSIDERAÇÕES FINAIS}

Dessa maneira, a Síndrome de Burnout necessita de atenção, pois está relacionada com a saúde mental do trabalhador gerando impactos negativos em sua vida pessoal e profissional. Logo, os profissionais de saúde que lidam com o estresse cotidiano devem ficar atentos aos primeiros sinais de alarme de seu adoecimento e buscar ajuda especializada quando necessário. Portanto, tem-se como relevância o reconhecimento precoce do perfil de profissionais que apresentam maior risco de desenvolver a SB e, com isso, intervir precocemente a fim de evitar a progressão da patologia. À vista disso, há necessidade de políticas públicas relacionadas com essa temática, desde a graduação, com grades curriculares que busquem abordar sobre a SB até a educação continuada nos serviços públicos/privados de saúde, a fim de combater essa problemática. Verifica-se que há necessidade de estudos com maiores espaços amostrais. 


\section{REFERÊNCIAS}

1. ALVARES MEM, et al. Síndrome de burnout entre profissionais de saúde nas unidades de terapia intensiva: um estudo transversal com base populacional. Rev. bras. ter. intensiva, 2020; 251-260.

2. ALVES ME. Síndrome de Burnout. Dissertação (Especialização em Psiquiatria) - Centro de Ciências Biológicas e da Saúde. Fundação Universitária Mário Martins, Porto Alegre, 2017; 26p.

3. BALDONEDO-MOSTEIRO M, et al. Síndrome de burnout em trabalhadores de enfermagem brasileiros e espanhóis. Revista Latino-Americana de Enfermagem, 2019; 27: e3192.

4. BARRETO GAA, et al. Condições de trabalho da enfermagem: uma revisão integrativa. Revista de Divulgação Científica Sena Aires, 2021; 10(1): 13-21.

5. BRAYDE DHS, COSTA TNM. Prevalência de Síndrome de Burnout em médicos residentes de um hospital de ensino. Revista Eletrônica Acervo Saúde, 2019; 24: e758.

6. CÂNDIDO J, SOUZA LR. Síndrome de Burnout: as novas formas de trabalho que adoecem. Psicologia.pt, 2017; 28: 1-12.

7. COSTA KNFM, et al. Qualidade de vida relacionada à saúde dos profissionais de enfermagem. Revista de Enfermagem UFPE on line, 2017; 11(Supl. 2): 881-889.

8. CRUZ SP, ABELLÁN MV. Desgaste profissional, estresse e satisfação por não trabalhar com o doente em um hospital universitário. Revista Latino-Americana de Enfermagem, 2015; 23(3): 543-552.

9. FAGUNDES PS. Síndrome de Burnout entre profissionais de saúde: uma revisão de literatura. Trabalho de Conclusão de Curso (Especialização em Saúde do Trabalhador). Universidade de Santa Cruz do Sul (UNISC), Santa Cruz do Sul, 2016; 20 p.

10. LIMA AS, et al. Análise da prevalência da síndrome de burnout em profissionais da atenção primária em saúde. Trabalho, Educação e Saúde, 2018; 16: 283-304.

11. MARQUES GLC, et al. Síndrome de burnout entre médicos plantonistas de unidades de terapia intensiva. Jornal Brasileiro de Psiquiatria, 2018; 67(3): 186-193.

12. MOREIRA HA, et al. Síndrome de Burnout em médicos: uma revisão sistemática. Revista Brasileira de Saúde Ocupacional, 2018; 43: 1-11.

13. PANTOJA FGB, et al. Avaliação do burnout em trabalhadores de um hospital universitário do município de Belém (PA). Saúde em Debate, 2017; 41: 200-214.

14. PÊGO FPL, PÊGO DR. Síndrome de Burnout. Rev. Bras Med Trab, 2016; 14(2): 171-176.

15. ROCHA APF, et al. A saúde e o trabalho de médicos de UTI neonatal: um estudo em hospital público no Rio de Janeiro. Physis: revista de saúde coletiva, 2015; 25: 843-862.

16. SANTOS JS, et al. Síndrome de Burnout em enfermeiros de Unidade de Terapia Intensiva: produção científica de Enfermagem. Revista Destaques Acadêmicos, 2018; 10(3): 190-198.

17. SILVA ABN, et al. Síndrome de Burnout em Profissionais de Enfermagem na Unidade de Terapia Intensiva. Revista de Ciências da Saúde Nova Esperança, 2016; 14(1): 79-86.

18. SILVA GN. (Re) conhecendo o estresse no trabalho: uma visão crítica. Gerais: Revista Interinstitucional de Psicologia, $2019 ; 12(1): 51-61$.

19. SILVA RAD, et al. Síndrome de Burnout: realidade dos fisioterapeutas intensivistas?. Fisioterapia e Pesquisa, 2018; 25(4): 388-394.

20. SILVA RC, et al. Síndrome de burnout entre os profissionais de enfermagem que atuam na assistência. Biológicas \& Saúde, 2015; 5(17): 23-35.

21. SILVA VF, et al. Fatores que influenciam no estresse ocupacional dos enfermeiros que atuam no Programa Saúde da Família. Revista Científica Interdisciplinar, 2015; 2(2): 162-179.

22. SILVEIRA ALP, et al. Síndrome de Burnout: consequências e implicações de uma realidade cada vez mais prevalente na vida dos profissionais de saúde. Rev Bras Med Trab, 2016; 14(3): 275-84.

23. TOMAZ HC, et al. Síndrome de Burnout e fatores associados em profissionais da Estratégia Saúde da Família. Interface (Botucatu), 2020; 24(Supl. 1): e190634.

24. TEIXEIRA GS, et al. Qualidade de vida no trabalho e estresse ocupacional da enfermagem em unidade de pronto atendimento. Texto \& Contexto-Enfermagem, 2019; 28: e20180298.

25. ZANATTA AB, LUCCA SR. Prevalência da síndrome de burnout em profissionais da saúde de um hospital oncohematológico infantil. Revista da Escola de Enfermagem da USP, 2015; 49(2): 0253-0258.

26. ZOMER FB, GOMES KM. Síndrome de burnout e estratégias de enfrentamento em profissionais de saúde: uma revisão não sistemática. Revista de Iniciação Científica, 2017; 15(1): 55-68. 